\title{
Long-term survival with good functional capacity in unoperated complex congenital heart defect with balanced systemic and pulmonary circulation
}

\author{
Maciej Haberka • Ewa Jastrzębska-Maj • \\ Małgorzata Biedroń • Leszek Szymański • \\ Jolanta Zuziak-Pruska $\cdot$ Zbigniew Gąsior
}

Received: 13 March 2014 / Accepted: 16 March 2014/Published online: 29 March 2014

(C) The Author(s) 2014. This article is published with open access at Springerlink.com

\begin{abstract}
A 46-year-old woman was admitted to our Department of Cardiology for stable exertional symptoms of heart failure (NYHA I) and distal mild cyanosis. The medical history revealed a complex congenital heart defect (double-inlet ventricle; DIV) diagnosed early at the infancy, but without palliative surgery or radical repair. The patient was leading a near normal life doing all the house work and working as a full-time physical worker. She had three healthy children delivered through a C-section without cardiovascular complications or significant symptoms progression. None of them was diagnosed with a heart defect. Physical examination revealed a blood pressure of $110 / 70 \mathrm{mmHg}$, heart rate $70 \mathrm{bpm}$, distal mild cyanosis (oxygen saturation $71 \%$ ), clubbed fingers, loud systolic heart murmur in the precordial area with a left parasternal thrill and only a mild right heart failure symptoms. Electrocardiogram showed a normal sinus rhythm without atrioventricular conduction disturbances. The major routine laboratory tests abnormalities were: $\mathrm{Hb}-19.85 \mathrm{~g} / \mathrm{dl}, \mathrm{Ht}-61.30 \%$, Fe-50 mg/dl and NT-proBNP-428 pg/ml. Moreover, a few months earlier she was diagnosed a hypothyroidism and a levothyroxine supplementation was introduced. The performed 6 min walk test was used to assess functional capacity
\end{abstract}

M. Haberka $(\bowtie)$ · E. Jastrzębska-Maj · M. Biedroń .

L. Szymański · Z. Gąsior

2nd Department of Cardiology, Medical University of Silesia,

Ziołowa 45/47, 40-635 Katowice, Poland

e-mail: mhaberka@op.pl

J. Zuziak-Pruska

Department of Diagnostic Imaging, Upper Silesian Medical

Katowice, Katowice, Poland showed a maximal distance of $440 \mathrm{~m}$ with a desaturation (75-51\%). Transthoracic echocardiography and cardiac magnetic resonance (CMR) imaging showed a double-inlet left ventricle of normal systolic function with unrestrictive ventricular septal defect and rudimentary right ventricle, d-transposition of great arteries. Both atrio-ventricular valves reveal only a mild regurgitation. There are also concordant atria with normal venous return separated with a residual interatrial septum and no pathology within an aortic arch. The most important for a long survival and balanced systemic and pulmonary circulation is that there is normal flow through the aortic valve without any obstruction and a moderate-to-severe pulmonary stenosis with supravalvular pulmonary stenosis, either (Fig. 1). The usual surgical approach in infants is a palliative shunt operation followed by one of the Fontan operation modifications. DIV is believed to carry a poor prognosis if unoperated with most patients dying before 16 y.o. [1]. Survival into adulthood without any surgical treatment is extremely rare and is found only in patients with a left ventricle-type of single ventricle and similar to the presented case combination of heart defects with normal systolic ventricle function and a moderate-to-severe pulmonary stenosis, which seems a crucial condition of balanced pulmonary circulation. The haemodynamic changes during pregnancy may usually significantly worsen cardiovascular symptoms even long time after a delivery. However, our patient denied significant progression of symptoms and there were no complications during or afterwards which suggest a perfectly balanced circulation. CMR provides most necessary information in the complex congenital heart defect, but a heart catheterization may add unique data on pulmonary artery pressure and pulmonary circulation. However the good functional capacity and only a mild cardiovascular symptoms in our patient encourage 


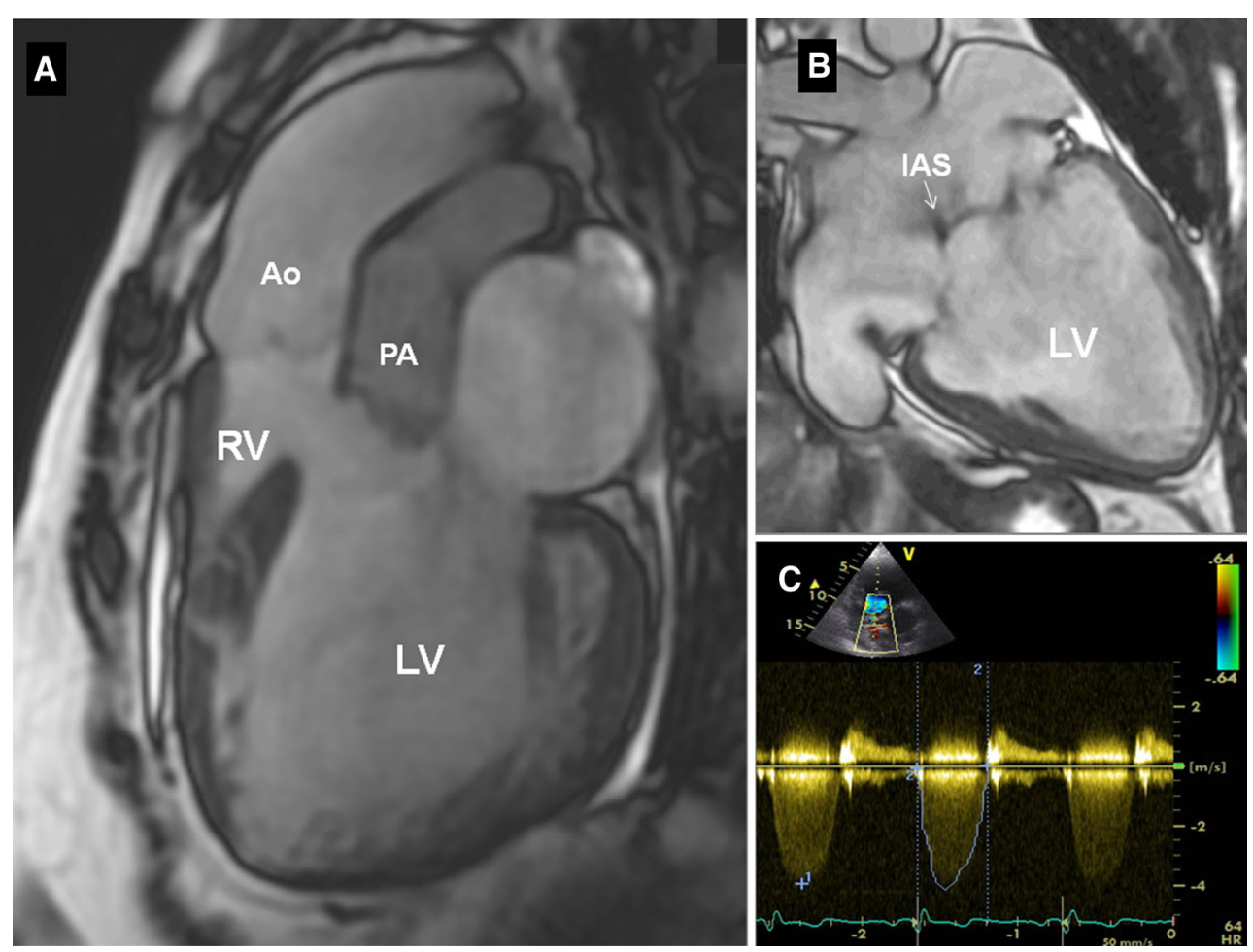

Fig. 1 Cardiac magnetic resonance $(\mathbf{a}, \mathbf{b})$ and transthoracic echocardiography (c) showing a left-type single ventricle $(L V)$, an unrestrictive interventricular septum defect with a rudimentary right ventricle

us to a strict follow-up for ventricular function, arrhythmia and functional capacity.

\section{Conflict of interest None.}

Open Access This article is distributed under the terms of the Creative Commons Attribution License which permits any use, distribution, and reproduction in any medium, provided the original author(s) and the source are credited.
$(R V)$, transposition of great arteries (Ao aorta; $P A$ pulmonary artery), residual interatrial septum $(I A S)$ (b) and a moderate-to-severe pulmonary stenosis in pulsed wave Doppler (c)

\section{Reference}

1. Moodie DS, Ritter DG, Tajik AJ, O'Fallon WM (1984) Long-term follow-up in the unoperated univentricular heart. Am J Cardiol 53:1124-1128 